\title{
Effects of Correct and Wrong Answers on ERPs Recorded under Conditions of the Continuous Performance Test in ADHD/Normal Participants
}

\author{
F. Ghassemi, ${ }^{1}$ M. H. Moradi, ${ }^{1}$ M. Tehrani-Doost, ${ }^{2,3}$ \\ and V. Abootalebi ${ }^{4}$
}

Neirofiziologiya/Neurophysiology, Vol. 42, No. 3, pp. 255-262, May-June, 2010.

\begin{abstract}
Received May 19, 2010.
Parameters of event-related potentials (ERPs) regarding correct and wrong answers under conditions of the continuous performance test (CPT) were measured in 50 adult subjects characterized by different levels of sustained attention with the absence/presence of attention deficit/hyperactivity disorders (ADHD). For ERP extraction, the average for each group of signals, which were time-locked to the onset of stimuli, was calculated; two ERP groups were considered separately for correct and wrong answers. In both groups, the P300 wave was clearly observed. The time dynamics of ERP components were investigated in six defined time blocks. At the peak of P300, a prominent component of brain activity could be observed. Some ERP morphological features (704 items) were extracted from these potentials. The results indicated that 11 of the obtained features showed a significant $(P<0.01)$ relation to the level of sustained attention. When comparing correct and wrong answers, 10 features in the normal group and 3 features in the ADHD group demonstrated significant differences $(P<0.05)$, which means that the participant's response is reflected in the features of EEG signal. The results reveal a promising relation between CPT results and some parameters of brain signals, which can be used for further evaluations of the sustained attention level.
\end{abstract}

Keywords: continuous performance test, event-related potentials, feature extraction, wave P300, sustained attention.

\section{INTRODUCTION}

The continuous performance test (CPT) $[1,2]$ is among the most popular tests for evaluating the level of sustained attention. Shifts of the latter are the core deficit in attention deficit/hyperactivity disorders (ADHD). Sustained attention is defined as the ability of a subject to maintain consistent behavioral responses during continuous and repetitive processing of stimuli whose non-arousing qualities would otherwise lead to habituation and distraction by other stimuli $[3,4]$.

Recording and analysis of event-related EEG potentials (ERPs) are an informative means for noninvasive monitoring of a few brain functions,

\footnotetext{
${ }^{1}$ Amirkabir University of Technology, Tehran, Iran.

${ }^{2}$ Tehran University of Medical Science, Tehran, Iran.

${ }^{3}$ Institute for Cognitive Science Studies (ICSS), Tehran, Iran.

${ }^{4}$ Department of Electrical and Computer Engineering, Yazd University, Yazd, Iran.

Correspondence should be addressed to M. H. Moradi

(e-mail: mhmoradi@aut.ac.ir).
}

including cognitive ones. Therefore, examination of these potentials is considered one of the adequate approaches in many neuroscience studies and clinical applications, in particular in the case of schizophrenia [5], dementia [6], and ADHD [7-9]. Results of a recent research [10] suggest that brain-based objective cognitive measures can support clinical decisions in the case of ADHD and improve the sensitivity and specificity of such decisions.

The wave P300 is an obvious cognitive component in ERPs, which may be achieved through an experimental oddball paradigm. Several studies have investigated cognitive ERPs and especially P300 in children diagnosed with ADHD. A decrement in the amplitude of P300 generated in response to both auditory and visual stimuli was reported for ADHDsuffering children $[8,11,14]$. The results obtained in ERP studies using different versions of the CPT confirmed the existence of such a decrement and indicated that adequate medication can lead to an increase in the amplitude of P300 [8]. 
Although a variety of studies was dedicated to the ERP components in ADHD children, only a few researches have investigated ADHD in adults. A study considering the level of sustained attention in normal adults during the CPT allowed an experimenter to investigate whether a central inhibitory mechanism intervenes to prevent the preparation and/or execution of a motor response. It was found that the N200 component was detectable in the no-go trials, while the P300 demonstrated specific scalp distributions related to the trial type [15]. Makeig et al. [16] studied early and late ERP components during the state of visual spatial attention. Their attempts resulted in the development of a robust and useful software for investigating EEG phenomena called EEGLAB $[17,18]$.

The approach we have pursued in our previous studies was to classify an adult population (including both ADHD and normal participants) according to three levels of attention. A promising accuracy (above $80 \%$ ) was acquired $[19,20]$, encouraging us to increase the number of classes for further studies. In this study, we examined the ERP components considering correct and wrong answers during the CPT within an adult population characterized by different levels of sustained attention. We also investigated the time dynamics of ERP components during different time blocks of the test.

\section{METHODS}

Participants. The examined group included 50 volunteers. The mean age of the participants was $29.78 \pm 6.15$ (mean \pm s.d) years. Twenty-six $(52 \%)$ subjects were women. All participants were examined for handedness using the Edinburgh test, and they, with no exception, were dextrals. They had normal or corrected-to-normal vision and were checked for color-blindness by the Ishihara test, which revealed that two participants were color-blind. According to the interview by a psychiatrist, ten drag-naive participants were diagnosed to have ADHD (inattention sub-type) based on the Diagnostic and Statistical Manual of Mental Disorders, 4th edition (DSM-IV) [21]. This diagnosis was confirmed by the results of the selfreport screening form of the Conners Adult ADHD Rating Scale (CAARS-S: SV) [22]. Other participants had no considerable psychiatric or medical disorders. The experiment was conducted in accordance with the Declaration of Helsinki. Also the review board of the Institute for Cognitive Science Studies (ICSS) revised and approved the experimental protocol. The main characteristics of the participants are summarized in Table 1.

CPT Task. The second version of the Conners' CPT [2] was used in this study, which is a "no-go" CPT task. Different letters of the English alphabet were presented randomly on the monitor screen, and participants were asked to click the left mouse button with the index finger of their dominant hand when any letter except the target " $X$ " appeared. Participants were instructed to respond as fast as they could, but also as accurately as possible. The six considerations of the proper protocol of the CPT were observed [2].

There were six blocks, with three sub-blocks in each containing 20 trials. Altogether, the experimental set involved 360 stimuli, namely $36 \mathrm{X}$ letters (no-go stimuli) and 324 other letters (go stimuli). The interstimulus intervals (ISIs) were 1,2 , or $4 \mathrm{sec}$ with a display time of $250 \mathrm{msec}$.

TABLE 1. Characteristics of the Participants

\begin{tabular}{|c|c|c|c|c|c|c|}
\hline $\begin{array}{l}\text { Level of sustained } \\
\text { attention }\end{array}$ & $\begin{array}{l}\text { Level } 1 \\
(n=10)\end{array}$ & $\begin{array}{l}\text { Level } 2 \\
(n=8)\end{array}$ & $\begin{array}{l}\text { Level } 3 \\
(n=9)\end{array}$ & $\begin{array}{l}\text { Level } 4 \\
(n=13)\end{array}$ & $\begin{array}{l}\text { Level } 5 \\
(n=10)\end{array}$ & $\begin{array}{l}\text { Total E } \\
(n=50)\end{array}$ \\
\hline \multirow[b]{2}{*}{ Age (mean \pm s.d), years } & $27.1 \pm 6.2$ & $31.5 \pm 5.7$ & $30 \pm 4.8$ & $30.6 \pm 6$ & $29.8 \pm 6.4$ & \multirow{3}{*}{$29.78 \pm 6.15$} \\
\hline & \multicolumn{4}{|c|}{$29.78 \pm 6.1$ (Normal) } & $\begin{array}{l}29.8 \pm 6.4 \\
\text { (ADHD) }\end{array}$ & \\
\hline \multirow{2}{*}{ Sex (women/men) } & $10 / 0$ & $7 / 1$ & $3 / 6$ & $1 / 12$ & $3 / 7$ & \\
\hline & \multicolumn{4}{|c|}{ 21/19 (Normal) } & 3/7 (ADHD) & 24.26 \\
\hline
\end{tabular}

Footnotes. The characteristics of 50 volunteers who participated in the test are shown. Level 1 includes participants with the best level of sustained attention, while level 5 corresponds to participants with the lowest attention level. The ADHD classification (level 5) is based on the psychiatrist's interview (according to DSM-IV) and confirmed by the CAARS-S: SV questionnaire. The normal classification is based on the CPT results. 
TABLE 2. Differences between the Parameters of ERP Components Related to Correct (C) and Wrong (W) Answers

\begin{tabular}{|c|c|c|c|c|c|c|c|c|c|c|c|c|}
\hline Group & Difference & $\mathrm{L}_{\mathrm{P} 3}$ & $\mathrm{~L}_{\mathrm{N} 2}$ & $\mathrm{~L}_{\mathrm{P} 32}$ & $\mathrm{~L}_{\mathrm{P} 3 \mathrm{~N} 2}$ & $\mathrm{~L}_{\mathrm{P} 2 \mathrm{~N} 2}$ & $\mathrm{~L}_{\mathrm{P} 3 \mathrm{~N} 4}$ & $\mathrm{~A}_{\mathrm{N} 2}$ & $\mathrm{~A}_{\mathrm{P} 4}$ & $\mathrm{~A}_{\mathrm{P} 34}$ & $\mathrm{~A}_{\mathrm{P} 2 \mathrm{~N} 2}$ & $\mathrm{~A}_{\mathrm{P} 4 \mathrm{~N} 4}$ \\
\hline ADHD & C-W & $63.5^{*}$ & -19.5 & $-68.5^{*}$ & $-44 *$ & 24.5 & -53.5 & 9.3 & -8.1 & 13.7 & -7.3 & -1.2 \\
\hline Normal & C-W & $-59 *$ & $-38.5^{*}$ & $-44.6^{*}$ & -20.5 & $24.1 *$ & $-57.5^{*}$ & $9.6^{*}$ & $-9 *$ & $13.4^{*}$ & $-10.5^{*}$ & $-7.2 *$ \\
\hline
\end{tabular}

Footnotes. L and A are the peak latency, msec, and amplitude, $\mu \mathrm{V}$. Some of the extracted features in correct and wrong answers are compared. Significantly different features are marked in the ADHD and normal groups by asterisks $(* P<0.05)$. Values indicate the differences $(\mathrm{CX}-\mathrm{WX})$ between the average of related parameters in correct and wrong answers.

The participants were seated on a comfortable chair with a place for relaxing the head. The test was performed in a quiet and dimly-lit room. The distance between the participant's eyes and a 19-inch monitor was $75 \pm 5 \mathrm{~cm}$ depending on the height of the tested person. The letters were $7.5 \mathrm{~cm}$ high and $7 \mathrm{~cm}$ wide, which resulted in a $7 \mathrm{deg}$ visual angle. They appeared white-colored on the black background. A short practice test $(70 \mathrm{sec})$ was performed before conducting the full test, to ensure that the participant has fully understood the task. Each test took approximately 14 min to complete. Twelve different measures are provided in the CPT II [2]:

Omissions: The number of non-targets to which the participant did not respond.

Commissions: The number of times the participant erroneously responded to the target ("X").

Hit Reaction Time (HRT): The mean response time for all non-X responses over all six time blocks.

HRT Standard Error (HRT s.e.): The standard error for the responses to non-X stimuli.

Variability of s.e.: The standard deviation of the 18 standard error values calculated for each sub-block.

Attentiveness $\left(d^{\prime}\right)$ : The difference between the signal (non-X) and noise $(\mathrm{X})$ distributions.

Perseveration: A response that occurs with a delay shorter than $100 \mathrm{msec}$ following presentation of the stimulus.

HRT Block Change: The slope of change in the reaction time over the six time blocks.
Hit s.e. Block Change: The slope of change in the reaction-time standard errors over the six time blocks.

HRT ISI Change: The slope of change in the reaction times over the three ISIs $(1,2$, and $4 \mathrm{sec})$.

Hit s.e. ISI Change: The slope of change in the reaction-time standard errors over the three ISIs.

In this study, the participants were classified according to five levels of attention. Level 1 was dedicated to the participants with the highest level of attention. Level 5 corresponded to the participants with ADHD, which was diagnosed by a psychiatrist based on DSM-IV classification and confirmed with the CAARS questionnaire. Normal participants were classified into four groups based on the results of the CPT interpreted by a specialist according to the CPT manual [2]. The characteristics of participants according to each level are summarized in Table 1.

EEG Recordings. The EEG activity was recorded by $19 \mathrm{Ag} / \mathrm{AgCl}$ electrodes mounted in an electrode cap and placed according to the international 10-20 standard. The impedance of all electrodes was kept below $5 \mathrm{k} \Omega$. The average of $\mathrm{A} 1$ and $\mathrm{A} 2$ was used as the reference. A bipolar vertical EOG was also recorded. Two additional bipolar channels were used for synchronization of the CPT system with EEG signals and recording responses of the participants' responses. A 32-channel AC/DC amplifier (Walter Graphtek, Germany) was used for data recording, and Pl-Winsor 3.0 software was used for data acquisition.

TABLE 3. Significant Correlated Features in Terms of Sustained Attention Level

\begin{tabular}{l|c|c|c|c|c|c}
\hline & $\mathrm{L}_{\mathrm{P} 4 \mathrm{~N} 4} \mathrm{CX}(\mathrm{B} 32)$ & $\mathrm{L}_{\mathrm{P} 3 \mathrm{~N} 4} \mathrm{WX}(\mathrm{B} 65)$ & $\mathrm{L}_{\mathrm{P} 32} \mathrm{WX}(\mathrm{B} 54)$ & $\mathrm{L}_{\mathrm{N} 4} \mathrm{WX}(\mathrm{B} 65)$ & $\mathrm{L}_{\mathrm{N} 2} \mathrm{WX}(\mathrm{B} 6)$ & $\mathrm{L}_{\mathrm{P} 2} \mathrm{WX}(\mathrm{B} 6)$ \\
\hline $\mathrm{R}$ & -0.4995 & -0.4116 & -0.4019 & 0.3872 & 0.3828 & 0.3828 \\
$\mathrm{P}$ value & 0.0002 & 0.003 & 0.0038 & 0.0055 & 0.0061 & 0.0061 \\
\hline \hline & $\mathrm{A}_{\mathrm{P} 3 \mathrm{~N} 4} \mathrm{WX}(\mathrm{B} 65)$ & $\mathrm{L}_{\mathrm{N} 4} \mathrm{WX}(\mathrm{B} 6)$ & $\mathrm{A}_{\mathrm{P} 2 \mathrm{~N} 2} \mathrm{WX}(\mathrm{B} 54)$ & $\mathrm{L}_{\mathrm{P} 3} \mathrm{WX}(\mathrm{B} 54)$ & $\mathrm{L}_{\mathrm{P} 4} \mathrm{WX}(\mathrm{B} 54)$ & \\
\hline $\mathrm{R}$ & 0.3805 & 0.3769 & -0.3736 & -0.3736 & -0.3702 \\
$\mathrm{P}$ value & 0.0064 & 0.007 & 0.0075 & 0.0075 & 0.0081 \\
\hline
\end{tabular}

Footnotes. The mentioned features demonstrated significant $(P<0.01)$ correlation with five levels of sustained attention. Correlation coefficients and $P$ values are indicated for the above features. 
The amplifier bandpass was $0.05-100 \mathrm{~Hz}$, and a 50 $\mathrm{Hz}$ notch filter was used for line noise reduction. The sampling rate was $200 \mathrm{sec}^{-1}$.

ERP Extraction. Data were analyzed using MATLAB 2009a software (MathWorks, USA). A suitable bandpass filter $(0.1$ to $85 \mathrm{~Hz})$ and a notch filter at $50 \mathrm{~Hz}$ were used to eliminate movement artifacts and to suppress the remaining line noise, respectively. The independent component analysis (ICA) was used for canceling EOG artifacts [23-25]. The ICA is referred to the separation of independent sources, which are mixed together with an unknown matrix, i.e., the mixing system and source signals are both unknown [26]. A combination of the efficient variant of fast ICA (EFICA) and efficient weightadjusted SOBI (EWASOBI) was chosen as the ICA method and realized using ICALAB software [27]. EOG components were automatically recognized by calculating the correlation between the recorded EOG and all achieved components by ICA. The component with a correlation coefficient greater than 0.8 and with the $\mathrm{P}$ value below 0.01 was determined as the EOG component. This component was eliminated, and then all other components were back-projected to their initial space.

For the epoch extraction, periods from $200 \mathrm{msec}$ before to $1000 \mathrm{msec}$ after the stimuli onset were considered. Four groups of ERPs were considered for each signal; these were potentials related to the target stimuli (X), non-target stimuli ( $\mathrm{nX}$ ), correct answers to the target stimuli (CX, which is the $\mathrm{X}$ that participant did not respond to), and wrong answers to the target stimuli (WX). Extracted epochs in each group, which were time-locked to the stimulus onset, were averaged to calculate the ERP parameters.

One of the main differences of sustained attention from other kinds of attention is the time factor. In order to investigate the ERP changes in time, six time windows were selected. Each window was $155 \mathrm{sec}$ long and contained three different ISI sub-blocks. The width of the window was calculated in such a way that all blocks contained the same number of targets. Therefore, each block included 6 target stimuli and 54 non-target ones for each participant.

Feature Extraction. The P300 wave (also known as $\mathrm{P} 3$, or P3b) is a large positive component that typically peaks about $300 \mathrm{msec}$ or more after the onset of a rare task-relevant stimulus [11-13]. The P300 window was selected at 300-650 msec after the stimulus onset $[11,12,28,29]$. Based on previous studies, the P300 demonstrates a centro-parietal scalp distribution that is maximal over the midline scalp sites $[11,12,28]$.
Thus, the Cz-channel signals were considered for the analysis in this paper. The amplitude of P300 was measured as the difference between the mean prestimulus baseline voltage and the largest positivegoing peak of the ERP waveform within the time window [12].

In addition to P300, four other components were defined and extracted from the ERPs observed. These were positive and negative peaks before and after the P300 called P200, N200, P400, and N400, respectively $[11,12,28-30]$.

The amplitude and latency were computed for these five components in each ERP group. Also, six relative components were defined by distracting the regarded values of two components (P34, P32, P3N2, P2N2, P4N4, and P3N4, where, e.g., P32 means P300-P200). These components were compared in ERPs related to correct and wrong answers. The differences were considered to be significant if the evaluated $\mathrm{P}$ values ( $t$-test) were smaller than 0.05 .

The defined features were calculated in the total signal, each of the six time blocks (B1 to B6) and nine relative blocks (B21, B32, B43, B54, B65, B31, B41, $\mathrm{B} 51$, and B61). Therefore, 704 features were obtained [2 parameters $\times 11$ components $\times 2(\mathrm{CX} / \mathrm{WX}$ ERP $) \times$ $\times 16$ blocks]. To investigate the relation between these features and CPT results, Pearson correlation was obtained between the level of sustained attention and the extracted ERP features.

\section{RESULTS}

Characteristics of the 50 participants are shown in Table 1. Considering only ADHD/normal classification, the mean age in the normal group was $29.78 \pm 6.15$ years, which practically coincided with that in the ADHD group. In the normal group, $47.5 \%$ of the population were men, while in the ADHD group the respective proportion was $70 \%$.

Four groups of ERPs (X, nX, CX, and WX) are illustrated in Fig. 1. This figure represents the ERPs for a typical participant in section A and grand average ERPs for all participants, all normal participants, and all ADHD participants in sections B, C, and D, respectively. The ERPs were smoothed with a $12 \mathrm{~Hz}$ low-pass filter. The detected P300 peaks are marked with filled circles. The amplitude and latency of the P300 wave in a grand average of X ERPs were $32 \mu \mathrm{V}$ and $435 \mathrm{msec}$, while the mean amplitude of P300 in $\mathrm{nX}$ ERPs was $10 \mu \mathrm{V}$. When comparing ERPs related to correct and wrong answers, the P300 amplitudes were 

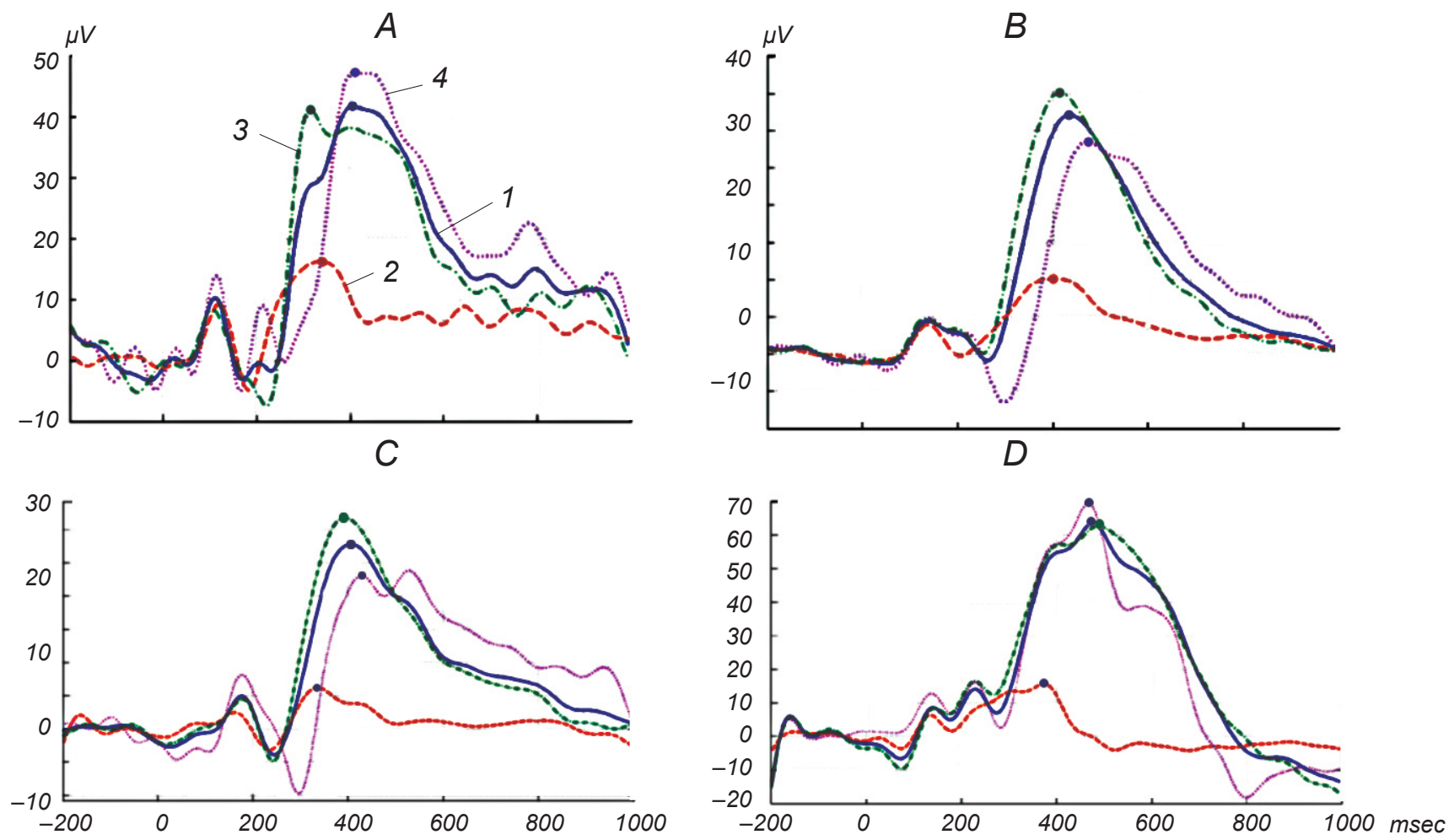

Fig. 1. Four different groups of ERPs (X, nX, CX, and WX, 1-4, respectively) are presented on distinct graphs for one typical participant (A), grand average of all participants (B), grand average of normal participants (C), and that of ADHD participants (D). The P300 peaks are marked with filled circles.

35 and $28.5 \mu \mathrm{V}$, respectively, while the values for the latencies were 415 and $475 \mathrm{msec}$.

Quantitative analysis of ERP variations in the groups related to correct and wrong answers was provided by performing the $t$-test on 22 extracted features from the ERP $(2$ parameters $\times 11$ components in the total signal). Table 2 compares some of the extracted features related to correct and wrong answers where ADHD and normal groups were considered separately. It should be noted that only values with significant differences $(P<0.05)$ in two groups are shown. Symbols "A" and "L" are used for the amplitude and latency, respectively. The mentioned subscript indicates the related absolute or relative component. For example, $\mathrm{L}_{\mathrm{P} 3 \mathrm{~N} 2}$ means a difference between the latencies of $\mathrm{P} 300$ and $\mathrm{N} 200$.

In order to study the P300 component variations with time, six time windows were considered. Figure 2 demonstrates the computed ERPs. In the first row, ERP groups, which were averaged for all 50 participants in each block, are shown. To investigate the time dynamics of the components, topomaps for the P300 peaks were provided. The middle row of Fig. 2 demonstrates the topomaps for grand-average CX ERP (correct answers to target stimuli) at the peak of the P300 component. At this moment, most of the scalp electrodes recorded an evident positivity. Topomaps for WX ERP (wrong answers to target stimuli, where the participant erroneously clicked to the presentation of $\mathrm{X}$ ) are demonstrated in the last row. Topomaps provided in this figure are created by EEGLAB software [34-35]. Although there were small changes between the blocks, a stable activity pattern could be observed in all blocks.

In the next step, Pearson correlations were calculated between the levels of sustained attention and defined ERP features. The $P$ values for 63 features were below 0.05 , while 11 of them, as indicated in Table 3, had $\mathrm{P}$ values smaller than 0.01 . The greatest correlation coefficient was -0.4995 . It belonged to $\mathrm{L}_{\mathrm{P} 4 \mathrm{~N} 4} \mathrm{CX}(\mathrm{B} 32)$, i.e., to the difference between the latencies of $\mathrm{P} 400$ and N400 in CX ERPs when comparing the third block with the second block. 

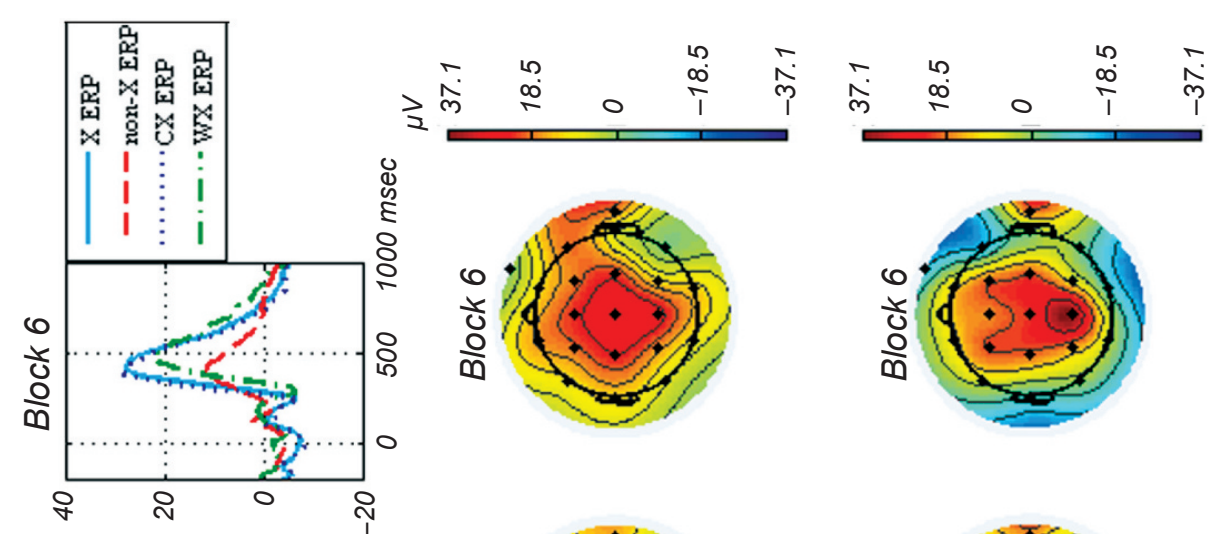

ت
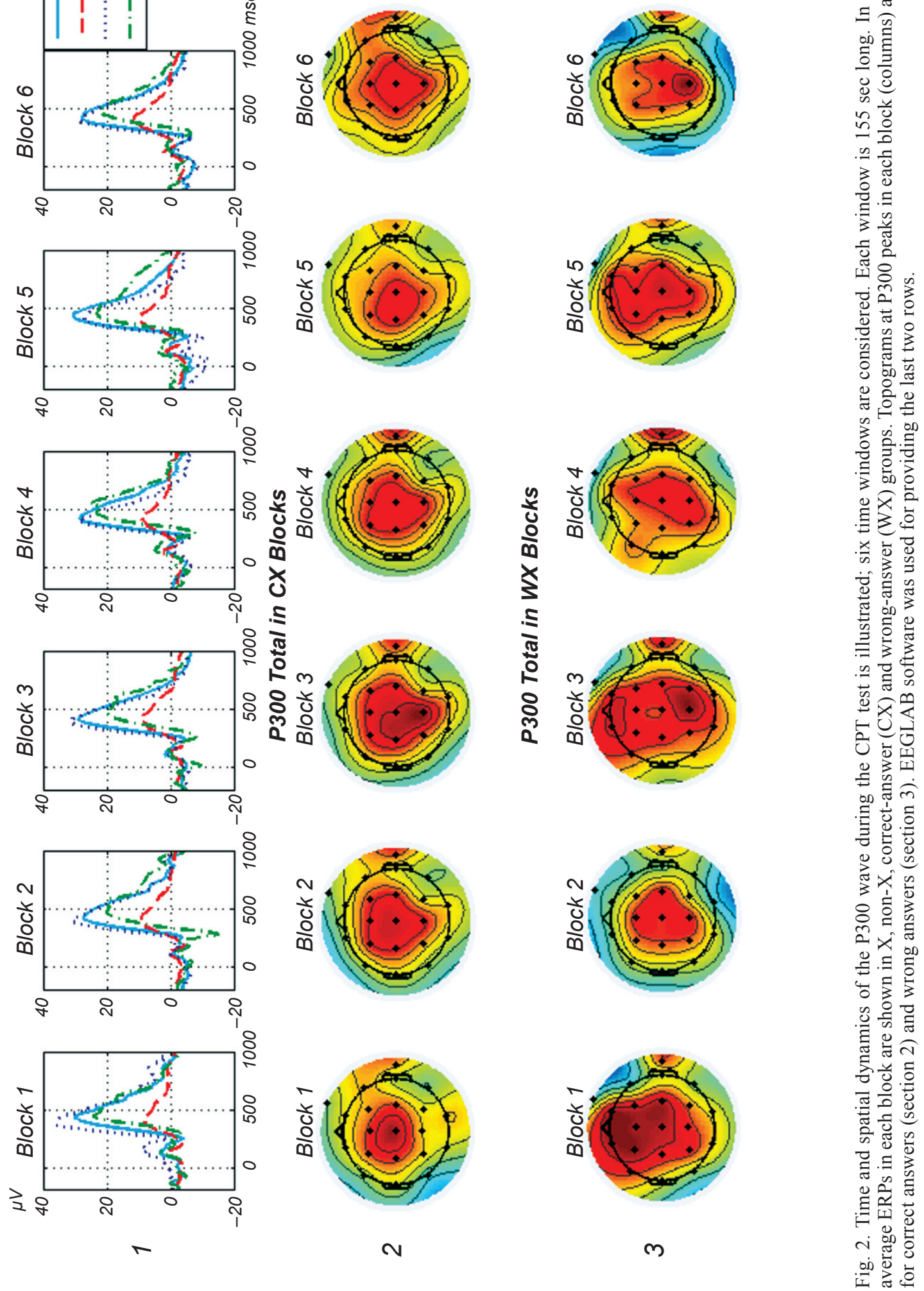


\section{DISCUSSION}

Figures $1 \mathrm{C}$ and $1 \mathrm{D}$ indicate that there is an obvious specificity of the P300 component for target stimuli (X ERP) compared to that for non-target stimuli (non-X ERP) in both normal and ADHD groups, which is in complete agreement with the data of previous studies [11-13, 19-20]. An interesting result is that the appearance of P300 for target stimuli was irrespective of the participant's answers. This finding was observed not only in the grand average ERP but also in every individual normal or ADHD participant, which could be due to the characteristics of the task and rareness of target stimuli caused by the proper ratio of target $v s$ total stimuli (1 to 10 ).

It is noteworthy that the peak of P300 in correctly answered targets in the normal group was greater in amplitude and shorter in latency compared to that related to the wrong answers. Investigating the ADHD group separately revealed that the latency of P300 was still shorter for correct answers, but the amplitude was smaller in comparison with that related to the wrong answers. The difference in the latency of P300 between the correct and wrong answers was significant $(\mathrm{P}<0.05)$ in both normal and ADHD groups.

Comparison of the ERPs in the groups of correct and wrong answers revealed that some features are significantly different in both normal and ADHD groups. This finding means that there is a significant difference in the ERP components of the participants between the correct and wrong answer groups.

For the normal group of subjects, five significant features were related to the amplitude, and five were related to the latency. The $\mathrm{L}_{\mathrm{P} 3}, \mathrm{~A}_{\mathrm{P} 4}, \mathrm{~L}_{\mathrm{P} 32}, \mathrm{~L}_{\mathrm{N} 2}, \mathrm{~A}_{\mathrm{P} 2 \mathrm{~N} 2}$, $\mathrm{A}_{\mathrm{P} 4 \mathrm{~N} 4}$, and $\mathrm{L}_{\mathrm{P} 3 \mathrm{~N} 4}$ were significantly larger in the wrong group of answers, while the $\mathrm{A}_{\mathrm{P} 34}, \mathrm{~A}_{\mathrm{N} 2}$, and $\mathrm{L}_{\mathrm{P} 2 \mathrm{~N} 2}$ were larger in correct answers. The largest difference in the average amplitude was $13.4 \mu \mathrm{V}$, and it was related to $\mathrm{A}_{\mathrm{P} 34}$, while the biggest difference in the average latency was $59 \mathrm{msec}$ for $\mathrm{L}_{\mathrm{P} 3}$.

For the ADHD group, only the differences in the latencies were significant. These features were all related to P300 (P3, P32 and P3N2). In all cases, the latency of this wave in wrong-answer ERPs was greater than in correct answers. The largest difference in the mean latency was $68.5 \mathrm{msec}$ for $\mathrm{L}_{\mathrm{P} 32}$.

In order to analyze the time dynamics of the components during a sustained attention task, the ERP parameters were calculated within different time blocks. The ERPs plotted in the first row of Fig. 2 were computed for electrode $\mathrm{Cz}$, and the manifested dynamics in the ERP could be traced in the $\mathrm{Cz}$ electrode of the provided topomaps in the middle and last rows of Fig. 2. At the peak of $\mathrm{P} 300$, a prominent component of the brain activity could be observed in both correctand wrong-answer groups. This activity lasts in all blocks in a stable good-shaped pattern, especially for correct answers, as is shown in Fig. 2.

Results of calculation of Pearson correlation revealed that there is a significant relation $(P<$ $<0.01)$ between the level of sustained attention and 11 extracted features of ERPs. The best feature correlation coefficient was -0.4995 , and it was related to the correct answers, while the other ten features were related to wrong answers. Nine of them were related to the latency where the first three of them were on relative components (distracting 2 components) and the other six were on absolute components. The two features related to the amplitude were both on relative components between positive and negative peaks.

Summarizing the above data and considerations, the components of ERPs regarding the correct and wrong answers have been evaluated and compared, while adult participants performed a sustained attentionrelated task. The patterns of cerebral activity at the P300 peak show that there is a good pseudosymmetric pattern of activity in the brain during the appearance of P300. Normal and ADHD groups were investigated separately; this comparison revealed that ten features in the normal group and three features in the ADHD group demonstrated significant differences $(P<0.05)$ depending on correct and wrong answers. The significant correlation between the CPT results and many of the extracted features validates the implementation of the measurement of ERP features for further studies of the sustained attention level.

Acknowledgments. The authors are thankful to the Institute for Cognitive Science Studies (ICSS) for providing the EEG laboratory for performing the tests, and to Dr. Anahita Khorrrami and Eng. Amin Mohammadian for their assistance in designing the protocol and conducting the test for some participants. The authors also thank all participants for their contribution to this study.

\section{REFERENCES}

1. H. E. Rosvold, A. F. Mirsky, I. Sarason, et al., "A continuous performance test of brain damage," J. Consult. Psychol., 20, 343-350 (1956).

2. C. K. Conners and MHS Staff, Conners' Continuous Performance Test II (CPT II) Ver. 5, Multi-Health Systems, Canada (2004).

3. E. Kandel, J. Schwartz, and T. Jessell, "Principles of neural science," in: Visual Attention," McGraw-Hill (2000). 
4. I. H. Robertson, T. Manly, J. Andrade, et al., "Oops!: Performance correlates of everyday attentional failures in traumatic brain injured and normal subjects," Neuropsychologia, 24, 636-647 (1997).

5. J. Todd, P. T. Michie, U. Schall, et al., "Deviant matters: duration, frequency, and intensity deviants reveal different patterns of mismatch negativity reduction in early and late schizophrenia," Biol. Psychiat., 63, 58-64 (2008).

6. E. J. Golob, R. Irimajiri, and A. Starr, "Auditory cortical activity in amnestic mild cognitive impairment: relationship to subtype and conversion to dementia," Brain, 130, 740752 (2007).

7. M. Arns, S. Ridder, U. Strehl, et al., "Efficacy of neurofeedback treatment in ADHD: the effects on inattention, impulsivity and hyperactivity: a metaanalysis," J. Clin. EEG Neurosci., 40, 180-190 (2009).

8. R. J. Barry, A. R. Clarke, R. McCarthy, et al., "Eventrelated potentials in two DSM-IV subtypes of attentiondeficit/hyperactivity disorder: an investigation using a combined modality auditory/visual oddball task," in: Attention-Deficit/Hyperactivity Disorder (AD/HD) and the Hyperkinetic Syndrome (HKS): Current Ideas and Ways Forward, R. D. Oades (ed.), Nova Publishers, New York (2006), pp. 229-247.

9. S. J. Johnstone, R. J. Barry, and J. W. Anderson, "Topographic distribution and developmental time course of auditory event-related potentials in two subtypes of attention-deficit hyperactivity disorder," Int. J. Psychophysiol., 42, 73-94 (2001).

10. L. M. Williams, D. F. Hermens, T. Thein, et al., "Using brain-based cognitive measures to support clinical decisions in ADHD," Pediat. Neurol., 42, 118-126 (2010).

11. C. C. Duncan, R. J. Barry, J. F. Connolly, et al., "Eventrelated potentials in clinical research: Guidelines for eliciting, recording, and quantifying mismatch negativity, P300, and N400," Clin. Neurophysiol., 120, 1883-1908 (2009).

12. J. Polich, "Updating P300: An integrative theory of P3a and P3b," Clin. Neurophysiol., 118, 2128-2148 (2007).

13. S. H. Pateland, and P. N. Azzam, "Characterization of N200 and P300: selected studies of the event-related potential," Int. J. Med. Sci., 2, 147-154 (2005).

14. R. J. Barry, S. J. Johnstone, and A. R. Clarke, "A review of electrophysiology in attention-deficit/hyperactivity disorder: II. Event-related potentials," Clin. Neurophysiol., 114, 184-198 (2003).

15. M. Butti, A. Pastori, A. Merzagora, et al., "Multimodal analysis of a sustained attention protocol: Continuous performance test assessed with near infrared spectroscopy and EEG," in: Proceedings of the 28th IEEE EMBS Annual International Conference, New York, USA (2006), pp. 1040-1043.
16. R. S. Huang, T. P. Jung, A. Delorme, et al., "Tonic and phasic brain dynamics during responses to simulated driving challenges," NeuroImage, 47, Suppl. 1, S103 (2009).

17. A. Delorme and S. Makeig, "EEGLAB: An open source toolbox for analysis of single-trial EEG dynamics including independent component analysis," J. Neurosci. Meth., 134, 9-21 (2004).

18. http://www.sccn.ucsd.edu/eeglab/

19. F. Ghassemi, M. H. Moradi, M. Tehrani-Doost, et al., "Classification of sustained attention level based on morphological features of EEG's independent components," in: IEEE/ICME International Conference on Complex Medical Engineering (CME 2009), Tempe (2009), pp. 1-6.

20. F. Ghassemi, M. H. Moradi, M. Tehrani-Doost, et al., "Combination of independent component analysis and feature extraction of erp for level classification of sustained attention," in: 4th International IEEE/EMBS Conference on Neural Engineering (NER), Antalya (2009), pp. 136139.

21. Up To Date 17.3 (2009).

22. C. K. Conners, D. Erhardt, and E. P. Sparrow, Conners' Adult ADHD Rating Scales (CAARS), Multi-Health Systems (2002).

23. A. Delorme, T. Sejnowski, and S. Makeig, "Enhanced detection of artifacts in EEG data using higherorder statistics and independent component analysis," NeuroImage, 34, 1443-1449 (2007).

24. S. Vorobyov and A. Cichocki, "Blind noise reduction for multisensory signals using ICA and subspace filtering, with application to EEG analysis," Biol. Cybern., 86, 293303 (2002).

25. N. P. Castellanos and V. A. Makarov, "Recovering EEG brain signals: Artifact suppression with wavelet enhanced independent component analysis," J. Neurosci. Methods, 158, 300-312 (2006).

26. A. Hyvarinen, J. Karhunen, and E. Oja, Independent Component Analysis-Theory and Applications, John Wiley \& Sons, London, New York (2001).

27. http://www.bsp.brain.riken.jp/ICALAB/

28. V. Abootalebi, M. H. Moradi, and M. Khalilzadeh, "A comparison of methods for ERP assessment in a P300based GKT,” Int. J. Psychophysiol., 62, 309-320 (2006).

29. L. Zordan, M. Sarlo, and F. Stablum, "ERP components activated by the GO! and WITHHOLD! conflict in the random sustained attention to response task," Brain Cogn., 66, 57-64 (2008).

30. A. A. Kovalenko and V. B. Pavlenko, "Emotional significance of the stimulus features of the personality as factors reflected in the pattern of evoked EEG potentials," Neurophysiology, 41, No. 4, 283-302 (2009). 